\title{
A Fully Conserved Minimal Adjustment Scheme with $(T, S)$ Coherency for Stabilization of Hydrographic Profiles
}

\author{
XIDONG WANG \\ Key Laboratory of Marine Environmental Information Technology, State Oceanic Administration, \\ National Marine Data and Information Service, Tianjin, China \\ PETER C. CHU \\ Naval Ocean Analysis and Prediction Laboratory, Department of Oceanography, \\ Naval Postgraduate School, Monterey, California \\ GuiJun Han, Wei Li, Xuefeng Zhang, AND Dong Li \\ Key Laboratory of Marine Environmental Information Technology, State Oceanic Administration, \\ National Marine Data and Information Service, Tianjin, China
}

(Manuscript received 16 February 2012, in final form 5 August 2012)

\begin{abstract}
A new, fully conserved minimal adjustment scheme with temperature and salinity $(T, S)$ coherency is presented for eliminating false static instability generated from analyzing and assimilating stable ocean $(T, S)$ profiles data, that is, from generalized averaging over purely observed data (data analysis) or over modeled/observed data (data assimilation). This approach consists of a variational method with (a) fully (heat, salt, and potential energy) conserved conditions, (b) minimal adjustment, and (c) $(T, S)$ coherency. Comparison with three existing schemes (minimal adjustment, conserved minimal adjustment, and convective adjustment) using observational profiles and a simple one-dimensional ocean mixed layer model shows the superiority of this new scheme.
\end{abstract}

\section{Introduction}

Analysis and assimilation of ocean temperature and salinity $(T, S)$ profile data are a generalized average over purely observed data (data analysis) or over modeledobserved data (data assimilation) (Chu et al. 2004). For example, data assimilation is intended to blend the modeled variable $\left(\mathbf{x}_{m}\right)$ with observational data $\left(\mathbf{y}_{o}\right)$ (Sun 1999):

$$
\mathbf{x}_{a}=\mathbf{x}_{m}+\mathbf{W} \cdot\left[\mathbf{y}_{o}-H\left(\mathbf{x}_{m}\right)\right],
$$

where $\mathbf{x}_{a}$ is the assimilated variable, $H$ is an operator that provides the model's estimate at the observational points, and $\mathbf{W}$ is the weight matrix that is determined by various data assimilation schemes such as optimal interpolation (e.g., Lozano et al. 1996), Kalman filtering (e.g., Galanis et al. 2006), and variational methods (e.g.,

Corresponding author address: Guijun Han, 93 Liuwei Rd., Hedong District, Tianjin 300171, China.

E-mail: cora@mail.nmdis.gov.cn
Tang and Kleeman 2004). Due to the nonuniform vertical distribution of the observational profile data and the high nonlinearity of the equation of state of seawater, a false static instability may be generated. For example, 10-day Jet Propulsion Laboratory (JPL) Estimating the Circulation and Climate of the Ocean (ECCO) $(T, S)$ fields centered on 31 December 2008 (download on 19 February 2009 from the JPL web site: http://ecco.jpl.nasa.gov/ external/) show that a considerable portion $(11.6 \%)$ of the profiles are statically unstable (Chu and Fan 2010a).

For discrete samples $\left(T_{k}, S_{k}\right)$ at depth $z_{k}$ (negative value), $k=1,2, \ldots, K$ ( $k$ increasing downward $)$, the density difference between two adjacent levels,

$$
\begin{aligned}
E_{k} & =\rho\left(S_{k+1}, T_{k+1}, z_{k}\right)-\rho\left(S_{k}, T_{k}, z_{k}\right), \\
k & =1,2, \ldots, K-1,
\end{aligned}
$$

is taken after one is adiabatically displaced to the depth of the other. Here, $E_{k}$ is defined as the static stability (Lynn and Reid 1968), $\rho\left(S_{k+1}, T_{k+1}, z_{k}\right)$ is the local potential density at depth $\left(z_{k+1}\right)$ that the water parcel 
would acquire if adiabatically brought to a reference depth $\left(z_{k}\right)$, and $\rho\left(S_{k}, T_{k}, z_{k}\right)$ is the in situ density to the depth of the upper of the two adjacent levels $\left(z_{k}\right)$. The density inversion is defined by the occurrence of negative values of $E_{k}$. The minimum static stability is represented by $E_{k}=0$. It is not always possible to reach zero exactly due to the precision limitations of the temperature and salinity values used (Locarnini et al. 2006). As a result, the minimum value for the static stability is given by

$$
E_{k} \geq E_{\min }, \quad k=1,2, \ldots, K,
$$

where $E_{\min }$ is the reference value for the minimum static stability, which is a user-defined, small positive value. If static instability occurs in observed or averaged hydrographic casts [i.e., (2) is not satisfied], it needs to be adjusted (Chu and Fan 2010a).

Let an unstable pair of hydrographic casts $\left[\left(T_{k}, S_{k}\right)\right.$, $k=1,2, \ldots, K]$ be represented by a $2 K$-dimensional vector:

$$
\mathrm{x}=\left(T_{1}, T_{2}, \ldots, T_{K}, S_{1}, S_{2}, \ldots, S_{K}\right) .
$$

An adjustment process is used to change the vector $\mathbf{x}$ into stable casts:

$$
\begin{aligned}
\mathbf{x}+\Delta \mathbf{x}= & \left(T_{1}+\Delta T_{1}, T_{2}+\Delta T_{2}, \ldots, T_{K}+\Delta T_{K},\right. \\
& \left.S_{1}+\Delta S_{1}, S_{2}+\Delta S_{2}, \ldots, S_{K}+\Delta S_{K}\right) .
\end{aligned}
$$

Ideally, three principles should be considered to determine $\Delta \mathbf{x}:$ 1) conservation of heat, salt, and potential energy; 2) minimal adjustment, that is, minimizing $\|\Delta \mathbf{x}\|$ subject to stabilizing the casts $\mathbf{x}+\Delta \mathbf{x}$; and 3) preservation of $(T, S)$ coherency. However, these principles are not fully used in the existing three types of schemes: (a) convective adjustment (CA), (b) minimal adjustment (MA), and (c) conserved minimal adjustment (CMA).

The CA schemes, often used in ocean modeling, are based on the same original idea (e.g., Bryan 1969; Yin and Sarachik 1994): whenever a water column is statically unstable, temperature and salinity are vertically adjusted to make the water column neutrally stable, with heat and salt conserved in the process. The adjustment takes an iterative approach. The iteration continues between all adjacent levels until the static instability is removed in the entire water column. Since the constraints are only the conservation of heat and salt, usually the adjustment $\|\Delta \mathbf{x}\|$ is relatively large (Chu and Fan 2010b).

The MA scheme originally proposed by Jackett and McDougall (1995) is employed to solve the problem:

$$
\begin{aligned}
& \text { Minimize }\|\Delta \mathbf{x}\|^{2} \equiv \Delta \mathbf{x}^{\mathrm{T}} \mathbf{I} \Delta \mathbf{x} \quad \text { subject to } \\
& \mathbf{A} \cdot(\mathbf{x}+\Delta \mathbf{x}) \geq E_{\min }^{2},
\end{aligned}
$$

where $\mathbf{I}$ is the identity matrix. The finite-difference approximation of stability $E_{k}$ becomes the inner product of the matrix $\mathbf{A}$ and the profile vector $\mathbf{x}+\Delta \mathbf{x}$. The matrix $\mathbf{A}$ depends on the solution $\Delta \mathbf{x}$ to the minimization problem (4), implying that the constraints in (4) are nonlinear.

Generally, the norm $\Delta \mathbf{x}^{\mathrm{T}} \mathbf{I} \Delta \mathbf{x}$ in (4) is not desirable because (a) it is not invariant under linear transformations and (b) it ignores the sources of the errors in temperature and salinity. A quadratic form, $\Delta \mathbf{x}^{\mathrm{T}} \mathbf{C} \Delta \mathbf{x}$, with $\mathbf{C}$ the inverse of an error covariance matrix is likely to be a better norm since it will yield a solution in which changes of trusted values tend to be smaller in size than the changes of unreliable values. Furthermore, the quadratic norm is a natural nondimensional measure, which can easily characterize the changes in temperature and salinity. However, the error covariance matrix $\mathbf{C}$ needs to be calculated before using this quadratic norm.

Usually, an iteration method is used in the MA method. Based on the MA scheme with some modifications, Locarnini et al. (2006) proposed a local iterative separated method to minimally adjust unstable temperature and salinity profiles. Obviously, the MA scheme does not require conservation of heat, salt, and potential energy, which may lead to errors in estimating the oceanic impact on global climate change patterns (Chu and Fan 2010a) since oceanic heat and salt transports play an important role.

The CMA scheme was developed by Chu and Fan (2010a) to solve (4) by requiring conservation of heat and salt. A set of well-posed combined $(K+1)$ linear and $(K-1)$ nonlinear algebraic equations for $\left\{\Delta T_{k}, \Delta S_{k}, k=1,2, \ldots\right.$, $K\}$ is established and solved using Newton's method. The CMA scheme has the three features: 1) conservation of heat and salt,2) removal of static instabilities with small $(T, S)$ adjustments, and 3) an analytical form. However, it does not conserve the potential energy and preserve the $T-S$ coherency since the salinity adjustment is assumed to be proportional to the temperature adjustment,

$$
\gamma_{k} \Delta S_{k}=-\Delta T_{k}
$$

with a single proportionality,

$$
\gamma_{k}=\gamma \equiv \frac{\max \left(T_{k}\right)-\min \left(T_{k}\right)}{\max \left(S_{k}\right)-\min \left(S_{k}\right)} .
$$

During the adjustment, the property of the water mass changes if the $T-S$ relation does not preserve, and the horizontal kinetic energy changes if the potential energy does not conserve. All of these changes are not real.

Thus, it is urgent to design a scheme that satisfies the three requirements: 1) conservation of heat, salt, and potential energy; 2) minimal adjustment; and 3) preservation of $(T, S)$ coherency. A variational adjustment 
(VA) scheme with satisfaction of all the three requirements is developed in this study. This scheme shows much better performance than the existing CA, MA, and CMA schemes. The rest of the paper is outlined as follows. Section 2 describes the VA scheme. Section 3 introduces the data used in this paper. Section 4 presents a comparison among the CA, MA, CMA, and VA schemes. Section 5 shows the evolution of an upper-ocean mixed layer with the four different schemes. Section 6 presents the conclusions.

\section{VA scheme}

Without knowing or calculating the error covariance matrix $\mathbf{C}$, minimization of the simple cost function

$$
J=\frac{1}{2} \Delta \mathbf{x}^{\mathrm{T}} \mathbf{I} \Delta \mathbf{x}
$$

is used. If the error covariance matrix $\mathbf{C}$ is given, the identity matrix $\mathbf{I}$ is replaced by $\mathbf{C}$. The minimization is conducted with the strong constrains of the static stability in Eq. (2), conservation of heat, salt, and potential energy,

$$
\int_{-H}^{0} \Delta T d z=0, \quad \int_{-H}^{0} \Delta S d z=0, \quad \int_{-H}^{0} g z \Delta \rho d z=0
$$

and preservation of the $T-S$ relationship. Here, $H$ is the maximum vertical extension of the profile data; $(\Delta T, \Delta S)$ and

$$
\Delta \rho=\beta \Delta S-\alpha \Delta T
$$

are the adjustments of $(T, S, \rho)$ at $z$. The conservation expressions in Eq. (7) can be discretized by

$$
\begin{aligned}
& \sum_{k=1}^{K-1} \frac{\left(\Delta T_{k}+\Delta T_{k+1}\right)}{2}\left(z_{k}-z_{k+1}\right)=0, \\
& \sum_{k=1}^{k-1} \frac{\left(\Delta S_{k}+\Delta S_{k+1}\right)}{2}\left(z_{k}-z_{k+1}\right)=0, \quad \text { and } \\
& \sum_{k=1}^{k-1} \frac{\left(\Delta \rho_{k}+\Delta \rho_{k+1}\right)}{2} z_{k}\left(z_{k}-z_{k+1}\right)=0,
\end{aligned}
$$

which are rearranged into

$$
\begin{gathered}
\sum_{k=1}^{K} a_{k} \Delta T_{k}=0, \quad \sum_{k=1}^{K} a_{k} \Delta S_{k}=0, \\
\alpha g \sum_{k=1}^{K} b_{k} \Delta T_{k}-\beta g \sum_{k=1}^{K} b_{k} \Delta S_{k}=0,
\end{gathered}
$$

with

$$
\begin{aligned}
& a_{1}=\frac{\left(z_{1}-z_{2}\right)}{2}, a_{2}=\frac{\left(z_{1}-z_{3}\right)}{2}, a_{3}=\frac{\left(z_{2}-z_{4}\right)}{2}, \ldots, a_{k-1}=\frac{\left(z_{k-2}-z_{k}\right)}{2}, a_{k}=\frac{\left(z_{k-1}-z_{k}\right)}{2} . \\
& b_{1}=\frac{z_{1}\left(z_{1}-z_{2}\right)}{2}, b_{2}=\frac{z_{2}\left(z_{1}-z_{3}\right)}{2}, b_{3}=\frac{z_{3}\left(z_{2}-z_{4}\right)}{2}, \ldots, b_{k-1}=\frac{z_{k-1}\left(z_{k-2}-z_{k}\right)}{2}, b_{k}=\frac{z_{k}\left(z_{k-1}-z_{k}\right)}{2} .
\end{aligned}
$$

The $T-S$ relation is obtained using locally weighted linear regression from $N$ surrounding casts $\left(T_{k}^{(j)}, S_{k}^{(j)}, j=\right.$ $1,2, \ldots, N)$ (Fox et al. 2002):

$$
\begin{aligned}
& S_{k}=\bar{S}_{k}+a_{k}\left(T_{k}-\bar{T}_{k}\right), \\
& a_{k}=\frac{\sum_{j=1}^{N} b_{j}\left(S_{k}^{(j)}-\bar{S}_{k}\right)\left(T_{k}^{(j)}-\bar{T}_{k}\right)}{\sum_{j=1}^{N} b_{j}\left(T_{k}^{(j)}-\bar{T}_{k}\right)^{2}}, \bar{S}_{k}=\frac{\sum_{j=1}^{N} b_{j} S_{k}^{(j)}}{\sum_{j=1}^{N} b_{j}}, \\
& \bar{T}_{k}=\frac{\sum_{j=1}^{N} b_{j} T_{k}^{(j)}}{\sum_{j=1}^{N} b_{j}},
\end{aligned}
$$

where $\bar{T}_{k}$ and $\bar{S}_{k}$ are the weighted averages at the current location where the $(T, S)$ cast is adjusted, $\left\{a_{k}\right\}$ are the regression coefficients calculated from the $N$ surrounding casts, and $\left\{b_{j}\right\}$ are the weights,

$$
b_{j}=\exp \left[-\frac{\left(x_{j}-x_{0}\right)^{2}}{L_{x}^{2}}-\frac{\left(y_{j}-y_{0}\right)^{2}}{L_{y}^{2}}\right],
$$

where $\left(x_{0}, y_{0}\right)$ and $\left(x_{j}, y_{j}\right)$ are the east-west and northsouth positions of the current and the $j$ th surrounding casts, respectively, and $\left(L_{x}, L_{y}\right)$ are the decorrelation scales in the $(x, y)$ direction (see Fox et al. 2002). Equation (12) can be rewritten as

$$
\Delta S_{k}-a_{k} \Delta T_{k}=\bar{S}_{k}+a_{k}\left(T_{k}-\bar{T}_{k}\right)-S_{k},
$$

which provides a salinity adjustment with a preserving $T-S$ relation. Equation (15) shows the major differences between the existing (CA, MA, CMA) schemes and the VA scheme. The existing schemes are fully local 
TABLE 1. The $W O A 98$ profiles for the $53.5^{\circ} \mathrm{S}, 171.5^{\circ} \mathrm{E}$ grid box before stabilization (from Locarnini et al. 2006, their Table B1).

\begin{tabular}{|c|c|c|c|c|c|c|}
\hline$k$ & Depth (m) & $T\left({ }^{\circ} \mathrm{C}\right)$ & $S(\mathrm{ppt})$ & $\rho\left(S_{k+1}, T_{k+1}, Z_{k}\right)\left(\mathrm{kg} \mathrm{m}^{-3}\right)$ & $\rho\left(S_{k}, T_{k}, Z_{k}\right)\left(\mathrm{kg} \mathrm{m}^{-3}\right)$ & $E_{k}\left(\mathrm{~kg} \mathrm{~m}^{-3}\right)$ \\
\hline 1 & 0 & 7.1667 & 34.4243 & 26.9476 & 26.9423 & 0.0054 \\
\hline 2 & 10 & 7.1489 & 34.4278 & 26.8982 & 26.9939 & $-0.0957 *$ \\
\hline 3 & 20 & 7.0465 & 34.2880 & 26.9529 & 26.9443 & 0.0085 \\
\hline 4 & 30 & 7.0050 & 34.2914 & 27.0104 & 26.9990 & 0.0114 \\
\hline 5 & 50 & 6.9686 & 34.2991 & 27.0967 & 27.1028 & $-0.0061 *$ \\
\hline 6 & 75 & 7.0604 & 34.3073 & 27.2406 & 27.2120 & 0.0286 \\
\hline 7 & 100 & 6.9753 & 34.3280 & 27.3892 & 27.3560 & 0.0332 \\
\hline 8 & 125 & 6.9218 & 34.3604 & 27.5164 & 27.5046 & 0.0117 \\
\hline 9 & 150 & 6.8919 & 34.3697 & 27.6000 & 27.6316 & $-0.0316^{*}$ \\
\hline 10 & 200 & 6.9363 & 34.3364 & 27.8123 & 27.8302 & $-0.0179 *$ \\
\hline 11 & 250 & 7.0962 & 34.3415 & 28.0295 & 28.0421 & $-0.0126^{*}$ \\
\hline 12 & 300 & 7.1622 & 34.3367 & 28.2684 & 28.2593 & 0.0092 \\
\hline 13 & 400 & 6.8275 & 34.2852 & 28.6664 & 28.7281 & $-0.0618^{*}$ \\
\hline 14 & 500 & 7.4001 & 34.3123 & 29.3699 & 29.1238 & 0.2461 \\
\hline 15 & 600 & 6.2133 & 34.4022 & 29.9386 & 29.8292 & 0.1094 \\
\hline 16 & 700 & 5.9186 & 34.4868 & 30.5869 & 30.3978 & 0.1891 \\
\hline 17 & 800 & 4.5426 & 34.4904 & 31.0754 & 31.0488 & 0.0266 \\
\hline 18 & 900 & 4.1263 & 34.4558 & 31.6539 & 31.5377 & 0.1162 \\
\hline 19 & 1000 & 3.3112 & 34.4755 & & 32.1176 & \\
\hline
\end{tabular}

* Static instability.

adjustments; that is, no surrounding $(T, S)$ casts are used. However, the VA scheme uses $N$ surrounding $(T, S)$ casts to preserve the $T-S$ coherency.

A successive quadratic programming method (Schittkowski 1986) is used to solve the general nonlinear optimization problem. When optimality is not achieved, a positive definite approximation of the Hessian is updated according to the modified Broyden-Fletcher-GoldfarbShanno method (Powell 1978).

\section{Data}

Evident false static instability was found in earlier versions of the World Ocean Atlas (WOA) such as more than $44 \%$ in 1982 version of the WOA (WOA82; Jackett and McDougall 1995) and around 3\% in WOA94 (Chu and Fan 2010a). The static instability disappears in recent versions of WOA due to the utilization of the modified MA scheme, which adjusts the $(T, S)$ profiles. An example as described in appendix B of Locarnini et al. (2006) is used for illustrating the capability of the scheme. The area chosen for this example is the $1^{\circ}$ latitudelongitude box centered at $53.5^{\circ} \mathrm{S}, 171.5^{\circ} \mathrm{E}$ from the 1998 version of the World Ocean Atlas (WOA98). The area of interest is on the New Zealand Plateau, with a bottom depth below $1000 \mathrm{~m}$ and above $1100 \mathrm{~m}$. The month is October, during the early austral summer. There is no temperature or salinity data within the chosen $1^{\circ}$ box. Thus, the objectively analyzed values in this $1^{\circ}$ box will be dependent on the seasonal objectively analyzed field and the data in nearby $1^{\circ}$ grid boxes. There is much more temperature data than salinity data on the New Zealand Plateau for October. This contributes to six small (on the order of $10^{-2} \mathrm{~kg} \mathrm{~m}^{-3}$ ) inversions in the local potential density field calculated from objectively analyzed temperature and salinity fields (Table 1 ). This $(T, S)$ cast is used for evaluating the performance of the VA scheme versus the existing CA, MA, and CMA schemes.

\section{Verification}

\section{a. Metrics for verification}

Several metrics are used to evaluate the magnitude of the $(T, S)$ adjustment and the conservation of heat, salt, and potential energy. Similar to Chu and Fan (2010b), the relative root-mean adjustment (RRMA) is used to identify the changes in temperature,

$$
\operatorname{RRMA}_{T}=\frac{\sqrt{\frac{1}{K} \sum_{k=1}^{K}\left(\Delta T_{k}\right)^{2}}}{\max \left(T_{k}\right)-\min \left(T_{k}\right)} ;
$$

salinity,

$$
\operatorname{RRMA}_{S}=\frac{\sqrt{\frac{1}{K} \sum_{k=1}^{K}\left(\Delta S_{k}\right)^{2}}}{\max \left(S_{k}\right)-\min \left(S_{k}\right)}
$$

and temperature-salinity, 

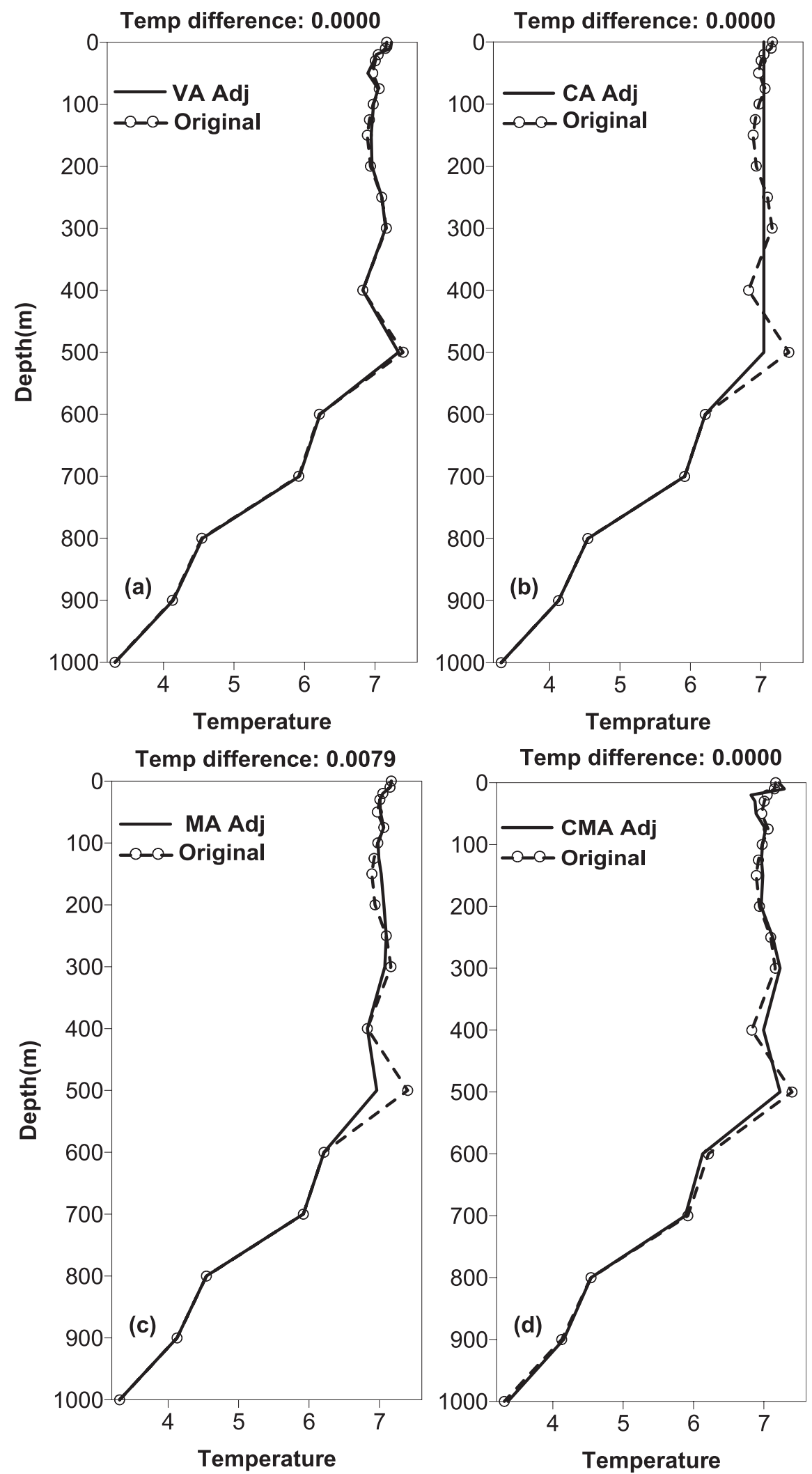

FIG. 1. Original (dashed) and adjusted (solid) temperature profiles $T_{k}$ at the $53.5^{\circ} \mathrm{S}, 171.5^{\circ} \mathrm{E}$ grid box using the (a) VA, (b) CA, (c) MA, and (d) CMA schemes. 
TABLE 2. RRMA ${ }_{T}$ and RRMA $S$ with the four adjust schemes on the original profile shown in Table 1. Here, the values for CA, MA, and CMA are from Chu and Fan (2010a).

\begin{tabular}{lcc}
\hline \hline Schemes & RRMA $_{T}$ & RRMA $_{S}$ \\
\hline CA & 0.0287 & 0.1905 \\
MA & 0.0220 & 0.0492 \\
CMA & 0.0241 & 0.0241 \\
VA & 0.0043 & 0.1192 \\
\hline
\end{tabular}

$$
\operatorname{RRMA}=\frac{\sqrt{\frac{1}{K} \sum_{k=1}^{K}\left(\Delta T_{k}\right)^{2}}}{\max \left(T_{k}\right)-\min \left(T_{k}\right)}+\frac{\sqrt{\frac{1}{K} \sum_{k=1}^{K}\left(\Delta S_{k}\right)^{2}}}{\max \left(S_{k}\right)-\min \left(S_{k}\right)} .
$$

is used as a metrics to identify the change of the adjusted from original casts. RRMA represents the mean adjustment relative to the range of a profile. The total heat, salt, and potential energy (PE) changes of the water column within this $1^{\circ} \times 1^{\circ}$ grid box are estimated by

$$
\begin{aligned}
\Delta Q & =A \rho_{0} c_{p} \int_{-H}^{0} \Delta T d z, \quad \Delta(\text { Salt })=A \int_{-H}^{0} \Delta S d z, \\
\Delta \mathrm{PE} & =\int_{-H}^{0} g z \Delta \rho d z
\end{aligned}
$$

where $\rho_{0}\left(=1028 \mathrm{~kg} \mathrm{~m}^{-3}\right)$ is the characteristic density, $c_{p}\left(=4002 \mathrm{~J} \mathrm{~kg}^{-1} \mathrm{~K}^{-1}\right)$ is the specific heat for the seawater, $H=1000 \mathrm{~m}$, and $A$ is the area of the grid box,

$$
A=\left(\frac{\pi}{180} R\right)^{2} \cos \varphi
$$

Here, $R(=6370 \mathrm{~km})$ is the earth radius, and $\varphi\left(=53.5^{\circ}\right)$ is the latitude of the grid box.

\section{b. Verification}

Figure 1 shows the comparison of temperature adjustments using the four schemes (CA, MA, CMA, and VA). The VA, CA, and CMA schemes conserve the heat, but the MA scheme does not. The three schemes with a minimum adjustment requirement (VA, MA, and CMA) generate the new profiles by basically following the original profile pattern, but the CA scheme does not. Among the three heat conserved schemes, the CA scheme has the largest change (0.0296) and the VA scheme has the smallest change (0.0043) (see Table 2). For the layer between 400 and $600 \mathrm{~m}$, the MA scheme has largest adjustment among VA, CA, MA, and CMA, which may lead to the largest $\mathrm{RRMA}_{T}$ while the other two schemes (VA and CMA) are nearly consistent with the original profile.
Figure 2 shows the comparison of salinity adjustment using the four schemes (CA, MA, CMA, and VA). The VA, CA, and CMA schemes conserve the salt, but the MA scheme does not. The three schemes with a minimum adjustment requirement (VA, MA, and CMA) generate the new profiles by basically following the original profile pattern, but the CA scheme does not. Among the three salt conserved schemes, the CA scheme has the largest change (0.1905), and the CMA scheme has the smallest change (0.0241), while the VA scheme has a modest change (0.1192) (see Table 2). Figure 3 shows the comparison of the static stability (i.e., $N^{2}$ ) adjustment using the four schemes (CA, MA, CMA, and VA). Among them, the CMA and MA schemes have small adjustments, and the VA and CA schemes have relatively large adjustments. It is also noted that salinity for the VA method is adjusted over the entire profile and the static stability increases at 800-m depth where the buoyancy frequency is near neutral (Fig. 3a). However, the salinity for the existing (CA, MA, and CMA) methods is adjusted above 600-m depth. This may be caused by the use of $N$ surrounding casts in the VA scheme [see Eq. (15)].

Table 3 lists the overall features of the adjustment using the four different schemes. The RRMA using VA (0.1235) is smaller than that using CA (0.2192), and larger than that using either MA (0.0712) or CMA (0.0482). The heat, salt, and potential energy are fully conserved using the VA scheme, but are not fully conserved using the other schemes. It causes a heat loss of $1.4 \times 10^{8} \mathrm{~J} \mathrm{~m}^{-2}$, a salt loss of $2.31 \mathrm{~kg} \mathrm{~m}^{-2}$, and a potential energy gain of $30700 \mathrm{~J} \mathrm{~m}^{-2}$ using the MA scheme, plus a potential energy gain of $4600 \mathrm{~J} \mathrm{~m}^{-2}$ using the CMA scheme, and a potential energy gain of $32800 \mathrm{~J} \mathrm{~m}^{-2}$ using the CA scheme. With the geostrophic adjustment, the potential energy can been consumed by either the production of kinetic energy or gravitational waves that travel to infinity, effectively carrying energy away from the region (Marotzke and Scott 1999). Thus, it is likely that the nonconservation of the potential energy during the adjustment may change the velocity field.

\section{Free convection}

\section{a. Theoretical limit}

Let us now consider free convection (no winds) in a resting ocean of constant stratification $N_{\text {th }}$ (subscript "th" for thermocline) with a uniform and widespread buoyancy loss $\left(B_{0}\right)$ at the surface. The response to widespread cooling is one in which relatively small convection cells (plumes) develop. Fluid parcels in contact with the surface will become dense and sink under gravity, driving 

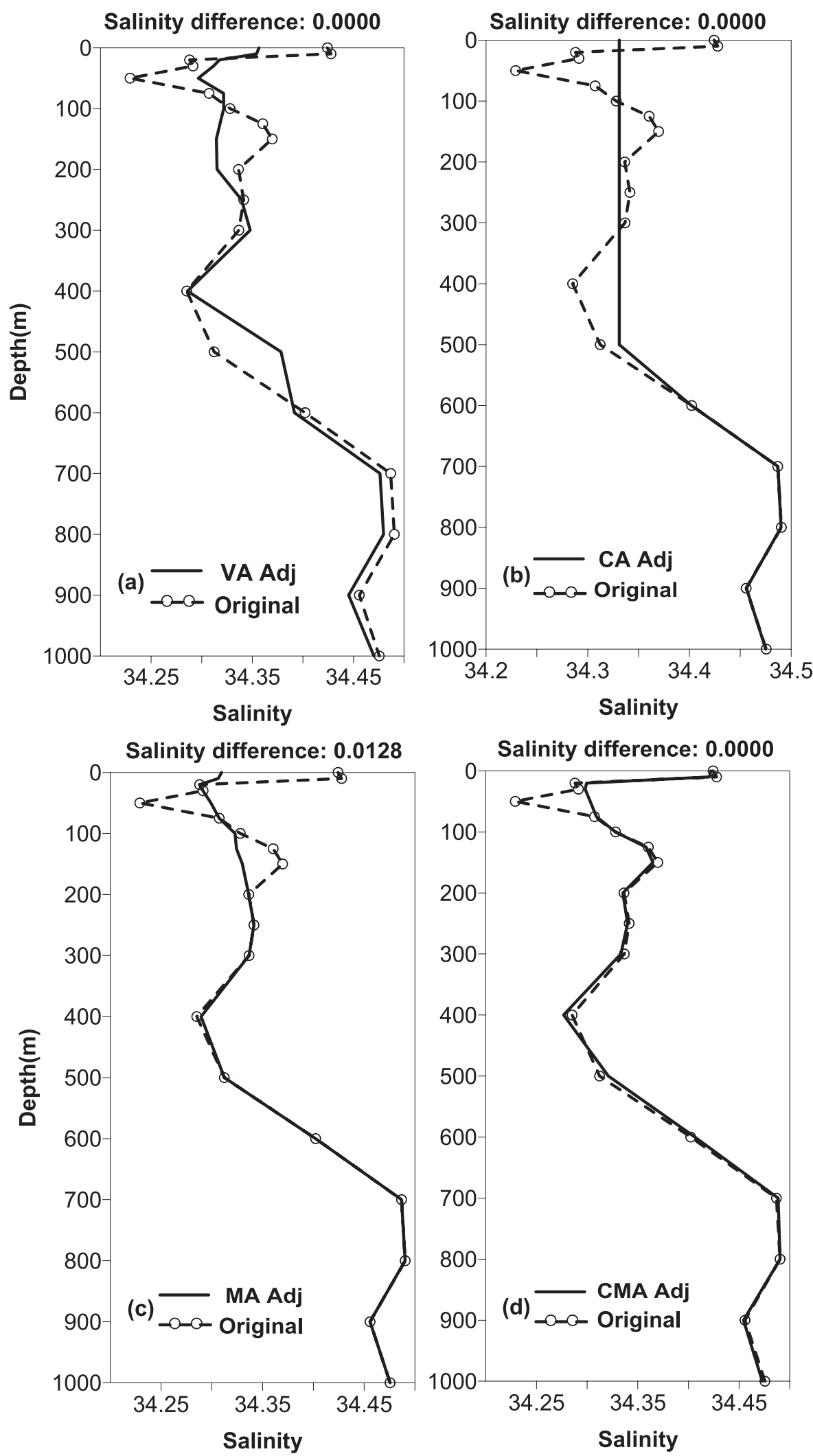

FIG. 2. Original (dashed) and adjusted (solid) salinity profiles $S_{k}$ at the $53.5^{\circ} \mathrm{S}, 171.5^{\circ} \mathrm{E}$ grid box using the (a) VA, (b) CA, (c) MA, and (d) CMA schemes. 

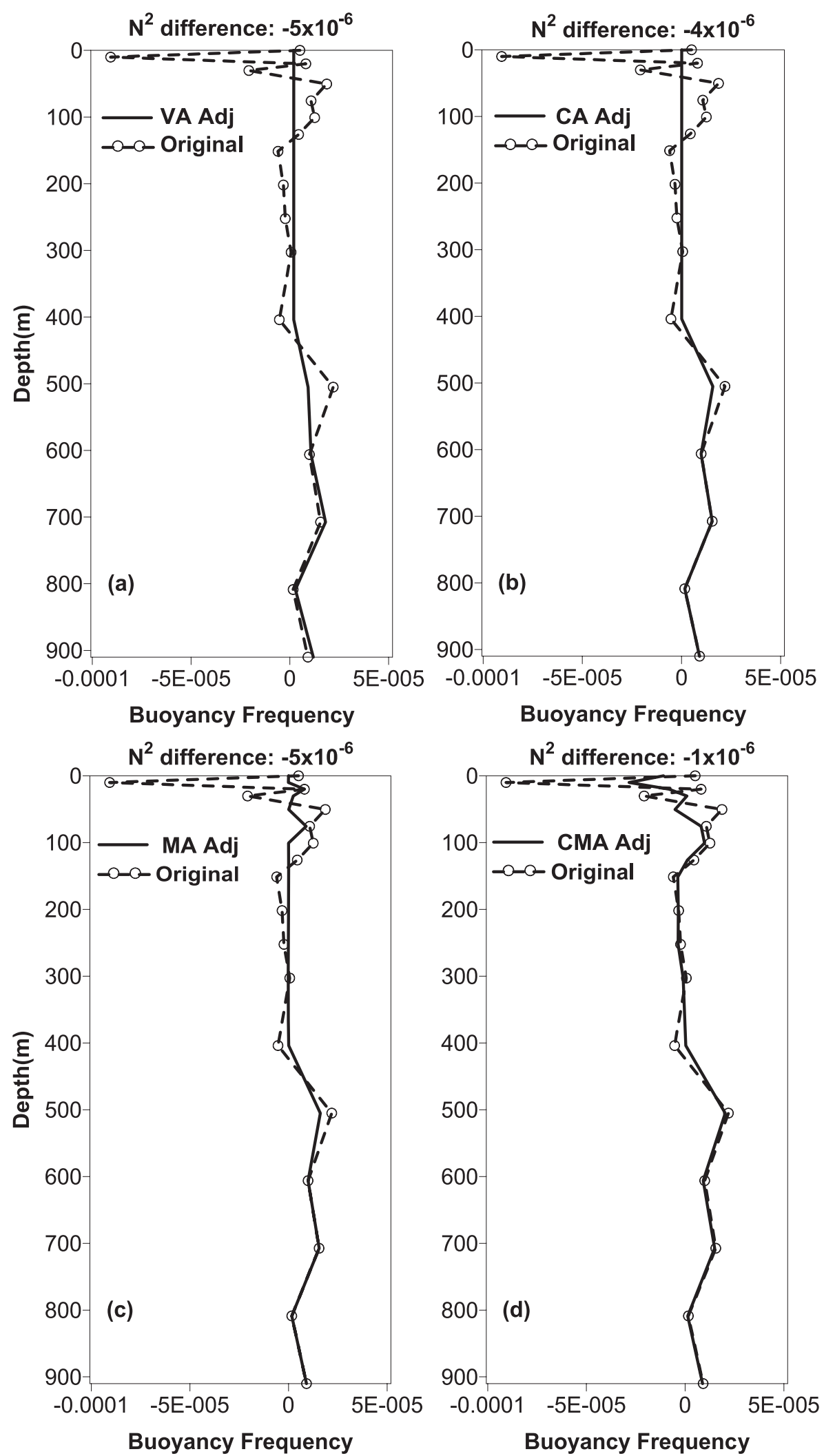

FIG. 3. Original (dashed) and adjusted (solid) $N^{2}$ profiles at the $53.5^{\circ} \mathrm{S}, 171.5^{\circ} \mathrm{E}$ grid box using the (a) VA, (b) CA, (c) MA, and (d) CMA schemes. 
TABLE 3. RRMA and changes in heat, salt, and potential energy using the four adjust schemes on the original profile shown in Table 1.

\begin{tabular}{lcccc}
\hline \hline & & $\begin{array}{c}\text { Heat change } \\
\text { per unit area } \\
\left(10^{8} \mathrm{~J} \mathrm{~m}^{-2}\right)\end{array}$ & $\begin{array}{c}\text { Salt change } \\
\text { per unit area } \\
\left(\mathrm{kg} \mathrm{m}^{-2}\right)\end{array}$ & $\begin{array}{c}\text { Potential energy } \\
\text { change per unit } \\
\text { area }\left(\mathrm{J} \mathrm{m}^{-2}\right)\end{array}$ \\
\hline CA & 0.2192 & 0 & 0 & 32800 \\
MA & 0.0712 & -1.4 & -2.31 & 30700 \\
CMA & 0.0482 & 0.0 & 0.0 & 4600 \\
VA & 0.1235 & 0.0 & 0.0 & 0.0 \\
\hline
\end{tabular}

the "free convective layer" below. Buoyancy is drawn upward, across the convective layer, offsetting its loss from the surface (Marshall and Schott 1999). For the same buoyancy loss at the ocean surface, the free convection depends on the temperature and salinity profiles, which are adjusted with various schemes (i.e., CA, MA, CMA, and VA). Thus, it is reasonable to use this process to investigate the impact of various adjustment schemes on the evolution of the free convective layer. If entrainment of stratified fluid from the base of the mixed layer can be neglected, the time evolution of the mixed layer depth (MLD) is calculated by (Turner 1973)

$$
h(t)=\frac{1}{N_{\mathrm{th}}} \sqrt{2 \int_{0}^{t} B_{0} d t .}
$$

If $B_{0}$ is constant in time, Eq. (18) becomes

$$
h(t)=\frac{1}{N_{\mathrm{th}}} \sqrt{2 B_{0} t} .
$$

In real oceans, the stratification below the mixed layer is not constant and the entrainment of stratified fluid from the base of the mixed layer cannot be neglected. The time evolution of MLD does not follow (18) or (19). Thus, numerical modeling is needed.

\section{b. Mixed layer modeling}

A one-dimensional mixed layer model (Price et al. 1994) is used to predict the evolution of the ocean mixed layer with free convection in a horizontally homogenous ocean. In this model, turbulent mixing occurrence involves three stability criteria. The vertical mixing is accounted for by static instability, and bulk and gradient Richardson number mixing. The critical bulk and gradient Richardson numbers are set to 0.65 and 0.25 , respectively. The model output includes the time evolution of the temperature and salinity profiles $[T(z, t), S(z, t)]$ as well as MLD $[h(t)]$.

\section{c. Impact of the schemes}

The five $(T, S)$ profile pairs (one original and four adjusted, shown in Figs. 1 and 2) do not have constant stratification in the thermocline. So, the theoretical limit in (18) or (19) might not be suitable for predicting $h(t)$. The mixed layer model (Price et al. 1994) is used to simulate free convection due to a steady heat loss of $220 \mathrm{~W} \mathrm{~m}^{-2}$ at the sea surface. The model is integrated for 5 days with the original and adjusted temperature and salinity profiles (by the four schemes) as the initial conditions.

Significant differences are found in the modelsimulated 5-day time evolution patterns of vertical temperature (Fig. 4) for the original and the four adjusted (CA, MA, CMA, and VA) profiles as the initial conditions. Hereafter, the adjusted profiles will be called the CA, MA, CMA, or VA profiles for convenience. The temperature field is similar in the mixed layer between using the original and MA profiles up to day 3.5 and different afterward with a vertically uniform layer (down to 500-m depth) for the MA profile and still stratified below $100 \mathrm{~m}$ for the original profile. The temperature field below the mixed layer is nearly $0.5^{\circ} \mathrm{C}$ colder for the MA profile than that for the original profile within about 3.5 days. At day 3.5, the thermocline suddenly breaks down for the MA profile, which contributes to the great mixed layer deepening. The temperature is similar in the mixed layer and there is little difference $\left(0.1^{\circ}-0.2^{\circ} \mathrm{C}\right)$ below the mixed layer between using the original and CMA profiles for the whole 5 days of simulation. The temperature is slightly warmer in the mixed layer using the VA profile than the original profile, but it is very similar below the mixed layer for the two profiles. Compared with the other three schemes and the original profile, using the CA profile reveals significant differences. The temperature in the upper $500 \mathrm{~m}$ throughout the region remains homogeneous without marked stratification.

The simulated time evolution patterns of MLD, $h(t)$, are very different among the original and four adjusted initial profiles (Fig. 5). The MLD increases linearly with almost the same rate between the CMA and the original profiles, although the MLD is $25 \mathrm{~m}$ thicker for the CMA profile. The MLD increases with time similarly when comparing the MA and VA profiles within day 1 and then very differently afterward. The MLD suddenly increases from 100 to $500 \mathrm{~m}$ at day 3.5 for the MA profile; however, the MLD increases to almost the same level as the theoretical limit (19) with a given $N_{\text {th }}(=1.414 \times$ $10^{-3} \mathrm{~s}^{-1}$ ) for the VA profile. This may indicate a positive feature of the VA scheme. For the CA profile, the initial MLD is deeper with about $500 \mathrm{~m}$, which may inhibit the mixing development due to the cooling. During 

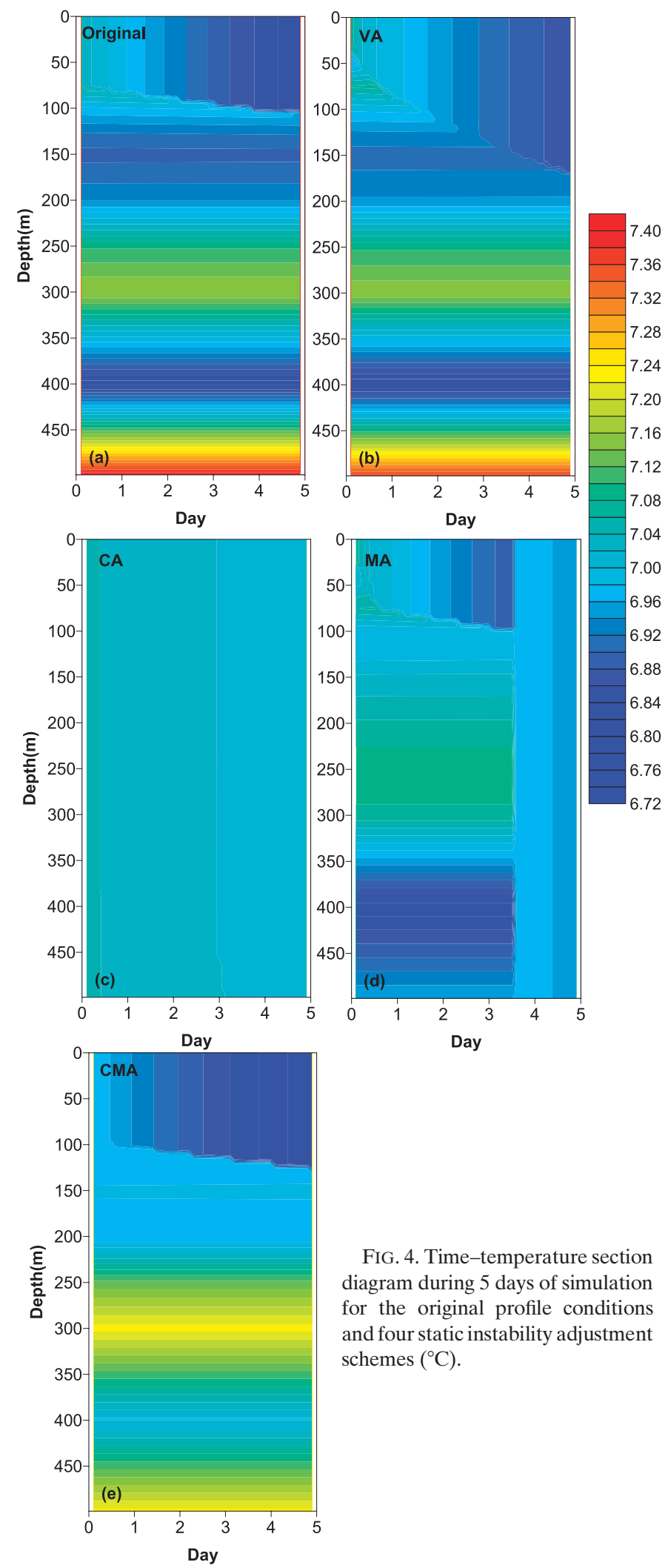

FIG. 4. Time-temperature section diagram during 5 days of simulation for the original profile conditions and four static instability adjustment schemes $\left({ }^{\circ} \mathrm{C}\right)$. 


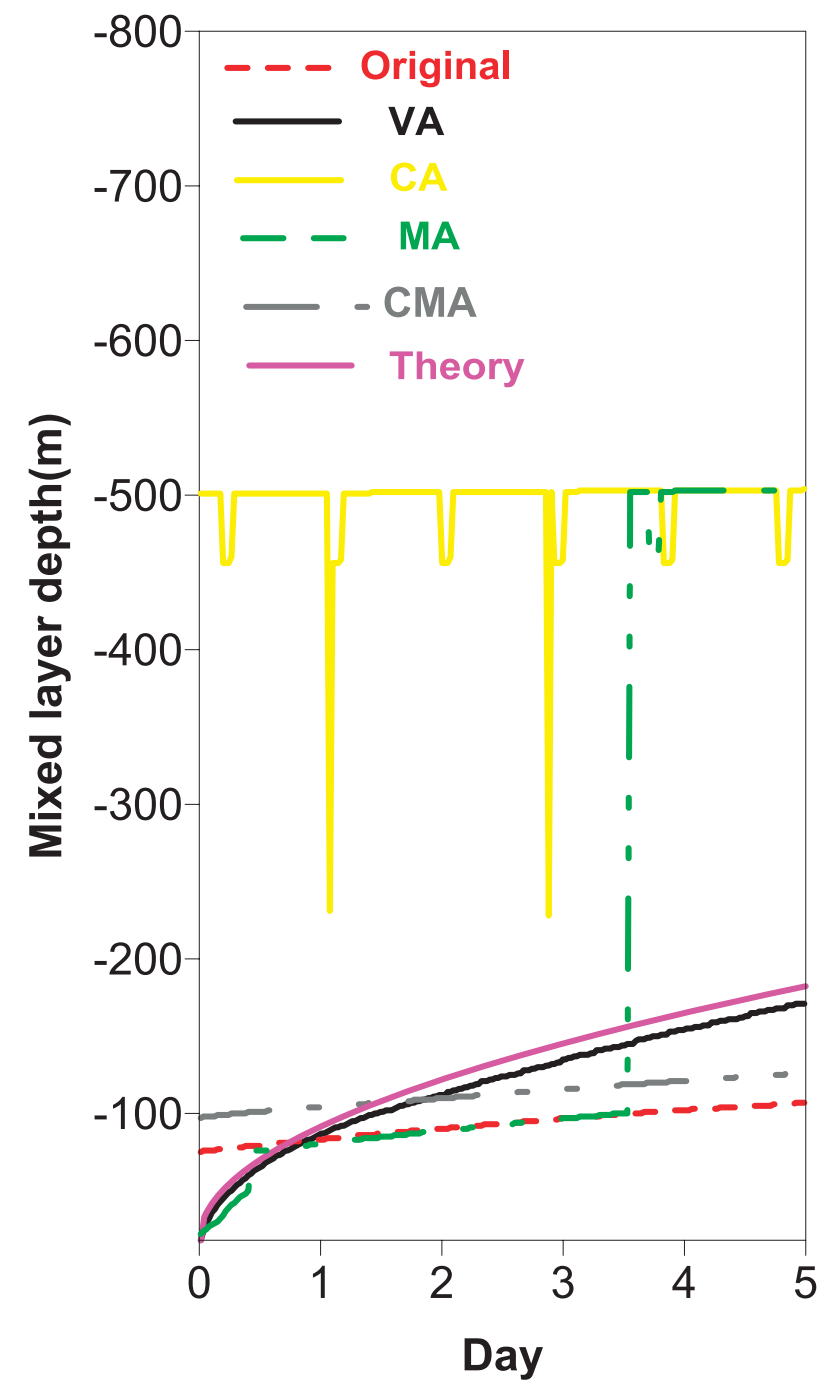

FIG. 5. The variability of mixed layer depth during 5 days of simulation for the original profile conditions and four adjustment schemes of static instability, as well as theory prediction.

5-day simulations, the MLD represents the evident oscillation of the 1-day period.

As described by Huang and Wang (2003), with a limited amount of heat loss due to cooling, a thin layer of water at the top of the mixed layer becomes heavier than the water below. This unstable stratification leads to a convective adjustment when gravitational potential energy is transformed into the small-scale energy, most of which is eventually dissipated into thermal energy. This energy loss is probably one of the most important components of the energetic balance in world oceans. Figure 6 shows the simulated time evolution of the potential energy per unit area relative to its initial value for the original and four adjusted profiles. For the original profile, it suddenly decreases about $500 \mathrm{~J} \mathrm{~m}^{-2}$ at the very beginning of the

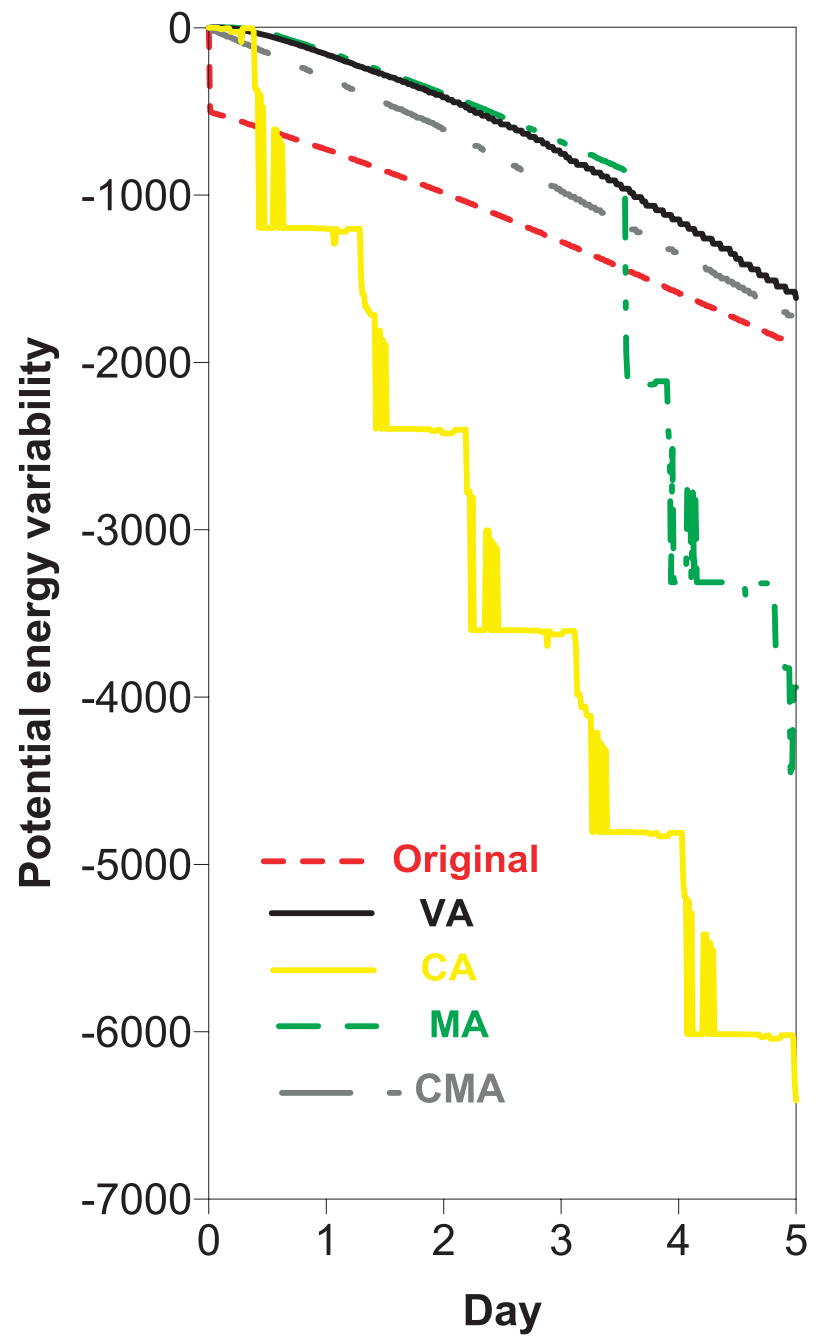

FIG. 6. The variability of gravitational potential energy per unit area relative to initial state during 5 days of simulation for the original profile conditions and four adjustment schemes of static instability $\left(\mathrm{J} \mathrm{m}^{-2}\right)$.

cycle due to the release of the static instability at the initial stage, and then decreases linearly to $1800 \mathrm{~J} \mathrm{~m}^{-2}$ at the end of day 5. For the MA profile, it decreases gradually within about 3.5 days, but very rapidly afterward increases to $3300 \mathrm{~J} \mathrm{~m}^{-2}$ on day 4. For both the CMA and VA profiles, it decreases gradually due to the surface cooling during the 5 days of simulation. After 5 days of free convection simulation, the potential energy reduction is $1615 \mathrm{~J} \mathrm{~m}^{-2}$ for the VA profile (minimum), $1739 \mathrm{~J} \mathrm{~m}^{-2}$ for the CMA profile, $1883 \mathrm{~J} \mathrm{~m}^{-2}$ for the original profile, $4500 \mathrm{~J} \mathrm{~m}^{-2}$ for the MA profile, and $6410 \mathrm{~J} \mathrm{~m}^{-2}$ for CA profile (maximum).

\section{Conclusions}

A new fully conserved minimal adjustment scheme is developed to eliminate the static instability of raw and 
averaged observational hydrographic data. This method adjusts the temperature and salinity profiles $\left\{\Delta T_{k}, \Delta S_{k}, k=\right.$ $1,2, \ldots, K\}$ simultaneously and efficiently on the base of three types of constraints: (a) heat, salt, and potential energy conservation; (b) a preserved $T-S$ relationship; and (c) the removal of static instability by minimal adjustment of the $T-S$ casts. With these constraints, a variational algorithm is designed to remove the false static instability induced by temperature-salinity data analysis/ assimilation.

The VA scheme can contribute to the data quality control process in data assimilations since it does not simply reject profiles with static instability. This method edits the profiles with the inequality constraint to satisfy static stability. Such a quality control process yields a reasonable response to a one-dimensional mixing model.

In principle, variational data assimilation can require the constraint conditions to satisfy static stability. These constraints suggested in the present paper can be forced directly to the cost function of the variational data assimilation in order to gain the assimilation result of static stability. The quadratic norm $\Delta \mathbf{x}^{\mathrm{T}} \mathbf{C} \Delta \mathbf{x}$ can be used to construct a rejection-acceptance criterion. With the features of (a) full (heat, salt, and potential energy) conservation, (b) minimal adjustment, and (c) $(T, S)$ coherency, the VA scheme can be used in global ocean $(T, S)$ data analysis and assimilation. However, further examination is needed since the results may vary with changing norms and since only limited data are used in this study for the verification.

Acknowledgments. This study is jointly supported by grants from the National Natural Science Foundation and Science and Technology Support Key Project Plan of China (Grants 41030854, 41176003, 41106005, 40906015, 40906016, and 2011BAC03B02). PCC is sponsored by the Naval Oceanographic Office.

\section{REFERENCES}

Bryan, K., 1969: A numerical method for study of the circulation of the World Ocean. J. Comput. Phys., 4, 347-376.

Chu, P. C., and C. W. Fan, 2010a: A conserved minimal adjustment scheme for stabilization of hydrographic. J. Atmos. Oceanic Technol., 27, 1072-1083.

$\ldots$, and $-2010 \mathrm{~b}$ : A fully conserved adjustment scheme for stabilization of hydrographic profiles. Preprints, 14th Symp. on Integrated Observing and Assimilation Systems for the
Atmosphere, Oceans, and Land Surface (IOAS-AOLS), Atlanta, GA, Amer. Meteor. Soc., 5B.1. [Available online at http://ams. confex.com/ams/pdfpapers/159655.pdf.]

_ G. G. Wang, and C. W. Fan, 2004: Evaluation of the U.S. Navy's Modular Ocean Data Assimilation System (MODAS) using the South China Sea Monsoon Experiment (SCSMEX) data. J. Oceanogr., 60, 1007-1021.

Fox, D. N., W. J. Teague, C. N. Berron, M. R. Carnes, and J. M. Lee, 2002: The Modular Ocean Data Assimilation System. J. Atmos. Oceanic Technol., 19, 240-252.

Galanis, G. N., P. Louka, P. Katsafados, G. Kallos, and I. Pytharoulis, 2006: Applications of Kalman filters based on non-linear functions to numerical weather predictions. Ann. Geophys., 24, 2451-2460.

Huang, R. X., and W. Wang, 2003: Gravitational potential energy sinks/sources in the ocean. Near Boundary Processes and Their Parameterization: Proc. 13th 'Aha Huliko'a Hawaiian Winter Workshop, Honolulu, HI, University of Hawaii at Manoa, 239-247.

Jackett, D. R., and T. J. McDougall, 1995: Minimal adjustment of hydrographic profiles to achieve static stability. J. Atmos. Oceanic Technol., 12, 381-389.

Locarnini, R. A., A. V. Mishonov, J. I. Antonov, T. P. Boyer, and H. E. Garcia, 2006: Temperature. Vol. 1, World Ocean Atlas 2005, NOAA Atlas NESDIS 61, $182 \mathrm{pp}$.

Lozano, C. J., A. R. Robinson, H. G. Arrango, A. Gangopadhyay, Q. Sloan, P. J. Haley, L. Anderson, and W. Leslie, 1996: An interdisplinary ocean prediction system: Assimilation strategies and structured data models. Modern Approaches to Data Assimilation in Ocean Modeling, P. Malanotte-Rizzoli, Ed., Elsevier, 413-452.

Lynn, R. G., and J. L. Reid, 1968: Characteristics and circulation of deep and abyssal waters. Deep-Sea Res., 15, 577-598.

Marotzke, J., and J. R. Scott, 1999: Convective mixing and the thermohaline circulation. J. Phys. Oceanogr., 29, 2962-2970.

Marshall, J., and F. Schott, 1999: Open-ocean convection: Observations, theory, and models. Rev. Geophys., 37, 1-64.

Powell, M. J. D., 1978: A fast algorithm for nonlinearly constrained optimization calculations. Numerical Analysis Proceedings, G. A. Watson, Ed., Lecture Notes in Mathematics, No. 630, Springer-Verlag, 144-157.

Price, J. F., T. B. Sanford, and G. Z. Forristall, 1994: Forced stage response to a moving hurricane. J. Phys. Oceanogr., 24, $233-$ 260.

Schittkowski, K., 1986: NLPQL: A FORTRAN subroutine solving constrained nonlinear programming problems. Ann. Oper. Res., 5, 485-500.

Sun, L. C., 1999: Data inter-operability driven by oceanic data assimilation needs. Mar. Technol. Soc. J., 33, 55-66.

Tang, Y., and R. Kleeman, 2004: SST assimilation experiments in a tropical Pacific Ocean model. J. Phys. Oceanogr., 34, 623642.

Turner, J. S., 1973: Buoyancy Effects in Fluids. Cambridge University Press, 368 pp.

Yin, F. L., and E. S. Sarachik, 1994: An efficient convective adjustment scheme for ocean general circulation models. J. Phys. Oceanogr., 24, 1425-1430. 